\title{
Coronary Heart Disease (CHD) in Elderly Patients: Which Drug to Choose, Ticagrelor and Clopidogrel? A Systematic Review and Meta-Analysis of Randomized Controlled Trials
}

\author{
Mohammed Ahmed Akkaif ${ }^{1}$, Abubakar Sha'aban ${ }^{1,2, * \mathbb{D}}$, Nur Aizati Athirah Daud ${ }^{1}$, Ismaeel Yunusa ${ }^{3}$, \\ Mei Li Ng ${ }^{4}$, Muhamad Ali Sk Abdul Kader ${ }^{5}$, Dzul Azri Mohamed Noor ${ }^{1}$ and Baharudin Ibrahim ${ }^{1,6, *}$ \\ 1 Discipline of Clinical Pharmacy, School of Pharmaceutical Sciences, Universiti Sains Malaysia, \\ Penang 11800, Malaysia; mohammed.alkaif@student.usm.my (M.A.A.); aizati@usm.my (N.A.A.D.); \\ dzulazri@usm.my (D.A.M.N.) \\ 2 Department of Clinical Pharmacy and Pharmacy Practice, Faculty of Pharmaceutical Sciences, \\ Ahmadu Bello University, Zaria 810107, Nigeria \\ 3 College of Pharmacy, University of South Carolina, Columbia, SC 29208, USA; iyunusa@mailbox.sc.edu \\ 4 Advanced Medical and Dental Institute, Universiti Sains Malaysia, Penang 13200, Malaysia; \\ nmeili07@nus.edu.sg \\ 5 Department of Cardiology, Penang General Hospital, Penang 10990, Malaysia; mdali_sheikh@hotmail.com \\ 6 Faculty of Pharmacy, University of Malaya, Kuala Lumpur 50603, Malaysia \\ * Correspondence: abuushaaban@usm.my (A.S.); baharudin.ibrahim@um.edu.my (B.I.); \\ Tel.: +60-1137188481 (A.S.); +60-103664181 (B.I.)
}

check for updates

Citation: Akkaif, M.A.; Sha'aban, A.; Daud, N.A.A.; Yunusa, I.; Ng, M.L.; Sk Abdul Kader, M.A.; Noor, D.A.M.; Ibrahim, B. Coronary Heart Disease (CHD) in Elderly Patients: Which Drug to Choose, Ticagrelor and Clopidogrel? A Systematic Review and Meta-Analysis of Randomized Controlled Trials. J. Cardiovasc. Dev. Dis. 2021, 8, 123. https://doi.org/ $10.3390 /$ jcdd 8100123

Received: 30 July 2021

Accepted: 24 September 2021

Published: 30 September 2021

Publisher's Note: MDPI stays neutral with regard to jurisdictional claims in published maps and institutional affiliations.

Copyright: (C) 2021 by the authors. Licensee MDPI, Basel, Switzerland. This article is an open access article distributed under the terms and conditions of the Creative Commons Attribution (CC BY) license (https:// creativecommons.org/licenses/by/ $4.0 /)$.

\begin{abstract}
Background: A new generation P2Y12 receptor inhibitor (ticagrelor) is recommended in current therapeutic guidelines to treat patients with coronary heart disease (CHD). However, it is unknown if ticagrelor is more effective than clopidogrel in elderly patients. Therefore, a systematic review was done to assess the effectiveness and safety of ticagrelor and clopidogrel in older patients with CHD to determine the appropriate antiplatelet treatment plan. Methodology: We performed a systematic review of randomized controlled trials (RCTs) to compare the effectiveness and safety of ticagrelor vs. clopidogrel in elderly patients with CHD. We selected eligible RCTs based on specified study criteria following a systematic search of PubMed and Scopus databases from January 2007 to May 2021. Primary efficacy outcomes assessed were major adverse cardiovascular events (MACEs), myocardial infarction (MI), stent thrombosis (ST), and all-cause death. The secondary outcome assessed was major bleeding events. We used RevMan 5.3 software to conduct a random-effects meta-analysis and estimated the pooled incidence and risk ratios (RRs) with $95 \%$ confidence intervals (CIs) for ticagrelor and clopidogrel. Results: Data from 6 RCTs comprising 21,827 elderly patients were extracted according to the eligibility criteria. There was no significant difference in the MACE outcome (incidence: $9.23 \%$ vs. $10.57 \%$; $\mathrm{RR}=0.95,95 \% \mathrm{CI}=0.70-1.28, p=0.72$ ), $\mathrm{MI}$ (incidence: $5.40 \%$ vs. $6.23 \%$; RR $=0.94,95 \% \mathrm{CI}=0.69-1.27, p=0.67$ ), ST (incidence: $2.33 \%$ vs. $3.17 \%$; RR $=0.61$, $95 \% \mathrm{CI}=0.32-1.17, p=0.13)$, and all-cause death $(4.29 \%$ vs. $5.33 \%$; $\mathrm{RR}=0.86,95 \% \mathrm{CI}=0.65-1.12$, $p=0.25$ ) for ticagrelor vs. clopidogrel, respectively. In addition, ticagrelor was not associated with a significant increase in the rate of major bleeding (incidence: $9.98 \%$ vs. $9.33 \%$ : RR $=1.37,95 \%$ $\mathrm{CI}=0.97-1.94, p=0.07)$ vs. clopidogrel. Conclusions: This study did not find evidence that ticagrelor is significantly more effective or safer than clopidogrel in elderly patients with CHD.
\end{abstract}

Keywords: ticagrelor; clopidogrel; systematic review; meta-analysis; randomized controlled trials

\section{Introduction}

The global burden of cardiovascular diseases has increased the global death rate, which has risen from 12.1 million (11.4-12.6 million) in 1990 to 18.6 million (17.1-19.7 million) in 2019 [1]. The incidence of CHD has also increased significantly [1]. CHD is a common condition associated with a high rate of sudden death and serious consequences, with 
almost 9 million fatalities worldwide in 2017, accounting for roughly $16 \%$ of all deaths [2]. Elderly patients account for a large proportion of those affected, far more than the enrolment rate in RCTs [3]. The risk score for recurrent ischemic events shows a three-fold higher event rate for an 80-year-old patient than for a 60-year-old [4]. The risk of bleeding was also increased significantly in elderly patients [5]. CHD also has imposed significant social and economic burdens all around the world. In recent years, the incidence of coronary heart disease has increased, especially in developing regions. Standardized management of $\mathrm{CHD}$ is the key to improving prognosis and reducing mortality [6]. Current guidelines for the management of patients with CHD recommend the use of dual antiplatelet therapy (DAPT) to reduce coronary thrombosis and mortality in patients who had acute coronary syndrome (ACS) or who have undergone percutaneous coronary intervention (PCI) [7]. For a long time, DAPT for patients with CHD has generally been aspirin combined with an adenosine diphosphate (ADP) receptor antagonist. Clopidogrel is the first choice for treatment, while ticagrelor is a relatively newer alternative [6]. PLATO trial in adult patients with ACS showed that ticagrelor was more effective than clopidogrel for 12 months [6,8]. Several recent literature reviews emphasized the importance of using ticagrelor as an alternative to clopidogrel to avoid clopidogrel resistance and improve clinical outcomes for some patients [9-11]. In addition, anti-platelet drugs increase the risk of bleeding especially in elderly patients $[6,12,13]$. Therefore, the choice of P2Y12 inhibitors should be carefully considered in this population. A recent randomized controlled trial (RCT) demonstrated the effect of platelet inhibitors in elderly patients with NSTE-ACS and observed no difference in ischemic events between potent P2Y12 inhibitors and clopidogrel, but noted a significant bleeding risk in potent P2Y12 inhibitors [14]. In addition, a recent observational study showed that the use of ticagrelor was associated with an increased risk of death and bleeding among elderly patients with myocardial infarction. Therefore, this study recommended the necessity of conducting a randomized study to examine the effects of ticagrelor on elderly patients [15]. Thus, the effect of ticagrelor vs. clopidogrel on ischemic and bleeding events was verified, but the results were different between studies. Therefore, we conducted a systematic review and meta-analysis to evaluate the outcomes of ticagrelor vs. clopidogrel therapy in elderly patients with CHD to provide more appropriate evidence for antiplatelet therapy in this population.

\section{Materials and Methods}

We conducted a meta-analysis of randomized controlled trials (RCTs) based on the Preferred Reporting Items for Systematic Review and Meta-Analysis (PRISMA) guideline [16]. The protocol was registered in PROSPERO (CRD42021257283).

\subsection{Search Strategy}

Relevant publications were searched from PubMed and Scopus from January 2007 to May 2021. The following keywords were used to find all publications related to this study: "ticagrelor OR AZD6140 and clopidogrel" and ("elderly OR old age patients") and ("acute coronary syndrome" OR "myocardial infarction" OR "unstable angina" OR "coronary heart disease" OR "coronary artery disease" OR "acute coronary syndrome" OR "myocardial infarction" OR "unstable angina"). Disagreements were settled by negotiating between two reviewers or consulting a third author. No language restrictions were enforced.

\subsection{Eligibility Criteria}

The following inclusion and exclusion criteria were used to evaluate eligible studies.

\subsubsection{Inclusion Criteria}

The type of studies included in this meta-analysis were RCTs with complete data. The study population was elderly patients (mean age $\geq 60$ years) with CHD, including those assigned to either ticagrelor or clopidogrel as secondary prevention after PCI with or without coronary stenting or after ACS admission. During the follow-up period following 
PCI, they reported clinical outcomes on effectiveness and safety as endpoints. The followup period used in the studies was 6-12 months.

\subsubsection{Exclusion Criteria}

Studies were excluded if they were non-randomized or poorly designed; animal experiments; had incomplete data or errors; repeated publications; studies without clinical outcomes of interest, editorials, reviews, case reports, commentary letters, economic evaluations; and studies that failed to provide the information required for this review.

\subsection{Outcome Assessment}

The primary outcomes are MACE, MI, ST, and all-cause deaths. MI was defined according to the American College of Cardiology Foundation/American Heart Association/European Society of Cardiology/World Heart Federation task force [17]. In addition, MACE includes cardiovascular death, stroke, and MI.

The secondary outcome was major bleeding for safety assessment, and its definition in the studies differ based on the Thrombolysis in Myocardial Infarction (TIMI) major bleeding, Platelet inhibition, and Patient Outcomes (PLATO) major bleeding, bleeding requiring transfusion or prolonging hospitalization, and International Society on Thrombosis and Haemostasis definitions [18]. In addition, for each study, the risk ratio (RR) was abstracted.

\subsection{Study Selection Process and Data Extraction}

We downloaded all references for consolidation, elimination of duplicates, and further analysis. First, title and abstract screening (Level 1) was done by two authors (M.A.A. and A.S.) independently to exclude documents that do not meet the inclusion criteria. Full text of articles that passed the Level 1 screening was then independently screened against the eligibility criteria (Level 2). Before deciding to include them, a third author (B.I.) was consulted to reach a decision and resolve inconsistencies in the literature. At least two reviewers then independently extracted data from eligible studies (Level 3).

\subsection{Risk of Bias Assessment}

The Cochrane Collaboration RoB 2.0 tool was used to assess the quality of all studies [19]. Two authors (M.A.A. and A.S.) independently assessed research quality based on the Cochrane Guide to Systematic Review; judgment, comparability, and outcomes were selected independently using the Cochrane Risk of Bias tool, and any disagreements were resolved unanimously.

\subsection{Statistical Analysis}

RevMan 5.3 statistical software was used for statistical analysis, including the risk ratio (RR) with 95\% confidence intervals (CI) [20].

As the studies were conducted in different geographic locations and at other times and populations composed of heterogeneous groups using different criteria for selection, statistical heterogeneity was examined using The Higgins I-square $\left(\mathrm{I}^{2}\right)$ statistic with a $p$-value to assess heterogeneity among studies using a random-effects model [21]. High heterogeneity was considered to be present when the $\mathrm{I}^{2}$ index was $>75 \%$ [21].

The publication bias was visually examined using funnel plots, but formal testing was not feasible due to the small number of studies [22]. Statistical significance for this meta-analysis was set at 0.05 .

Sensitivity analysis was performed using the "leave-1-out" method, excluding the studies one by one; for example, the Gimbel et al., (2020) study was excluded, and it is noted if there are any differences obtained in the results [23]. 


\section{Results}

\subsection{Study Selection}

The search primarily identified 6366 articles (after duplicates were removed) that were potentially relevant to the study. With the removal of review articles and after reviewing the title and abstract, 23 articles were assessed. According to the eligibility criteria, a total of 6 articles published between 2009 and 2020 were included in this study (Figure 1).

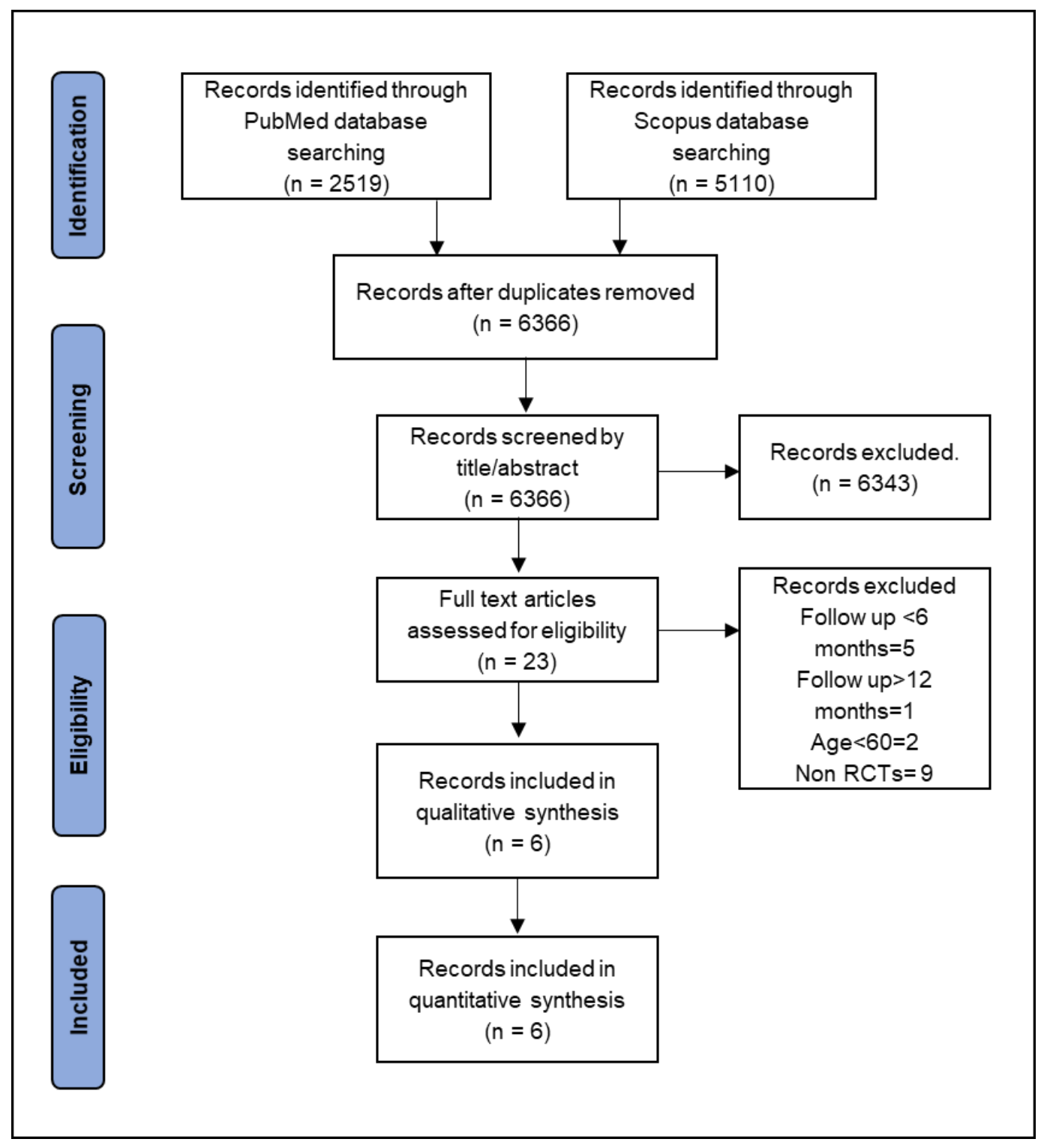

Figure 1. Flow chart of articles screening.

\subsection{Characteristics of Studies}

The study characteristics of the research publications and samples are included in Table 1. Detailed information about patient characteristics can be found in Table 2. All trials included were generally considered to be of a low bias risk based on the RoB2 tool of the Cochrane (Figure 2). All the six RCTs included [6,14,24-27] compared two groups, making a total of 10,936 cases in the ticagrelor group and 10,891 cases in the clopidogrel group. The mean age of the patients included in the six studies was 68.6 years old, with a follow-up of 12 months. 
Table 1. Characteristics of every included study.

\begin{tabular}{|c|c|c|c|c|c|c|c|c|c|c|}
\hline \multirow[t]{2}{*}{ Authors } & \multirow[t]{2}{*}{ Location } & \multirow{2}{*}{$\begin{array}{l}\text { Centres } \\
\text { (N.) }\end{array}$} & \multirow[t]{2}{*}{ Diagnosis } & \multirow{2}{*}{$\begin{array}{l}\text { Design } \\
\text { of the } \\
\text { Study }\end{array}$} & \multicolumn{2}{|c|}{$\begin{array}{c}\text { Age } \\
\text { Mean (SD) or Median } \\
\text { (IQR) }\end{array}$} & \multirow{2}{*}{$\begin{array}{c}\text { Follow } \\
\text { Up } \\
\text { (Months) }\end{array}$} & \multirow[t]{2}{*}{$\begin{array}{l}\text { Bleeding } \\
\text { Classification }\end{array}$} & \multicolumn{2}{|c|}{ Outcome Indication } \\
\hline & & & & & Ticagrelor & Clopidogrel & & & Ticagrelor & Clopidogrel \\
\hline $\begin{array}{l}\text { Gimbel et al., } \\
2020 \text { [14] }\end{array}$ & Netherlands & 12 & NSTE-ACS & RCTs & $\begin{array}{c}77.00 \\
(73-82)\end{array}$ & $\begin{array}{c}77.00 \\
(73-81)\end{array}$ & 12 & PLATO & $\begin{array}{l}\text { MACE, MI, } \\
\text { Mortality, } \\
\text { Bleeding }\end{array}$ & $\begin{array}{l}\text { MACE, MI, } \\
\text { ST, Mortality, } \\
\text { Bleeding }\end{array}$ \\
\hline $\begin{array}{l}\text { Goto et al., } \\
2015 \text { [27] }\end{array}$ & Japan & 110 & ACS & RCTs & $\begin{array}{c}67.00 \\
(12)\end{array}$ & $\begin{array}{c}66.00 \\
(11)\end{array}$ & 12 & PLATO & $\begin{array}{l}\text { MACE, MI, } \\
\text { Mortality, } \\
\text { Bleeding }\end{array}$ & $\begin{array}{l}\text { MACE, MI, } \\
\text { Mortality, } \\
\text { Bleeding }\end{array}$ \\
\hline $\begin{array}{l}\text { Park et al., } \\
2019 \text { [26] }\end{array}$ & Korea & 10 & ACS & RCTs & $\begin{array}{l}62.50 \\
(11.3)\end{array}$ & $\begin{array}{l}62.30 \\
(11.5)\end{array}$ & 12 & PLATO & $\begin{array}{l}\text { MACE, MI, } \\
\text { ST, Mortality, } \\
\text { Bleeding }\end{array}$ & $\begin{array}{l}\text { MACE, MI, } \\
\text { ST, Mortality, } \\
\text { Bleeding }\end{array}$ \\
\hline $\begin{array}{l}\text { Tang et al., } \\
2016 \text { [25] }\end{array}$ & China & 2 & STEMI & RCTs & $\begin{array}{l}64.36 \\
(11.4)\end{array}$ & $\begin{array}{l}64.18 \\
(11.1)\end{array}$ & 6 & TIMI & $\begin{array}{l}\text { MACE, } \\
\text { Mortality, } \\
\text { Bleeding }\end{array}$ & $\begin{array}{l}\text { MACE, MI, } \\
\text { ST, Mortality, } \\
\text { Bleeding }\end{array}$ \\
\hline $\begin{array}{l}\text { Wallentin } \\
\text { et al., } 2009 \text { [6] }\end{array}$ & USA & 862 & ACS & RCTs & 62.00 & 62.00 & 12 & PLATO & $\begin{array}{l}\text { MACE, MI, } \\
\text { ST, Mortality, } \\
\text { Bleeding }\end{array}$ & $\begin{array}{l}\text { MACE, MI, } \\
\text { ST, Mortality, } \\
\text { Bleeding }\end{array}$ \\
\hline $\begin{array}{c}\text { Wang and } \\
\text { Wang } 2016 \\
{[24]}\end{array}$ & China & 1 & ACS & RCTs & $\begin{array}{c}79.00 \\
(76-85)\end{array}$ & $\begin{array}{c}80.00 \\
(74-86)\end{array}$ & 12 & PLATO & $\begin{array}{l}\text { MACE, MI, } \\
\text { ST, Mortality, } \\
\text { Bleeding }\end{array}$ & $\begin{array}{l}\text { MACE, MI, } \\
\text { Mortality, } \\
\text { Bleeding }\end{array}$ \\
\hline
\end{tabular}

T-Ticagrelor; C-Clopidogrel; N.-number; MACE-major adverse cardiac events; MI-Myocardial infarction; ST-stent thrombosis; NSTE-ACS—non-ST-elevation acute coronary syndrome; STEMI—ST-elevation myocardial infraction; RCTs—randomized controlled trials; SD—standard deviation; IQR—interquartile range.

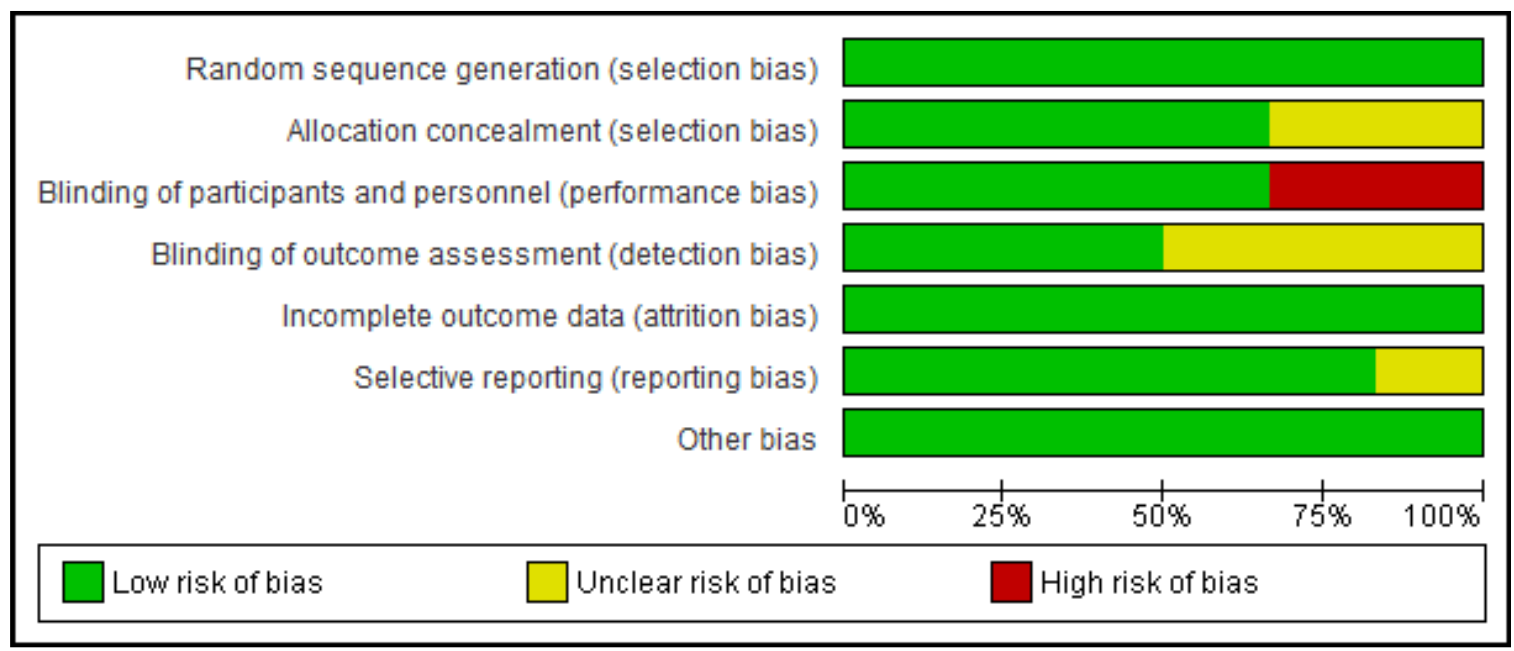

Figure 2. Graph of risk of bias. 
Table 2. Baseline characteristics of patients in each trial.

\begin{tabular}{|c|c|c|c|c|c|c|c|c|c|c|c|c|}
\hline \multirow[b]{2}{*}{ Medication } & \multicolumn{2}{|c|}{ Gimbel et al., 2020 [14] } & \multicolumn{2}{|c|}{ Goto et al., 2015 [27] } & \multicolumn{2}{|c|}{ Park et al., 2019 [26] } & \multicolumn{2}{|c|}{ Tang et al., 2016 [25] } & \multicolumn{2}{|c|}{$\begin{array}{l}\text { Wallentin et al., } \\
2009[6]\end{array}$} & \multicolumn{2}{|c|}{ Wang and Wang 2016 [24] } \\
\hline & Ticagrelor & Clopidogrel & Ticagrelor & Clopidogrel & Ticagrelor & Clopidogrel & Ticagrelor & Clopidogrel & Ticagrelor & Clopidogrel & Ticagrelor & Clopidogrel \\
\hline Loading dose (mg) & 180 & 300,600 & 180 & 300 & 180 & 600 & 180 & 600 & 180 & 300 & 180 & 300 \\
\hline Maintenance dose (mg) & 90 & 75 & 90 & 75 & 90 & 75 & 90 & 75 & 90 & 75 & 90 & 75 \\
\hline Patient number & 502 & 500 & 401 & 400 & 400 & 400 & 200 & 200 & 9333 & 9291 & 100 & 100 \\
\hline Male (\%) & $325(65)$ & $313(63)$ & ND & ND & $297(74.2)$ & $302(75.5)$ & $142(71)$ & $146(73)$ & $\mathrm{ND}$ & ND & ND & ND \\
\hline Female (\%) & $177(35)$ & $187(37)$ & $95(23.7)$ & $93(23.3)$ & ND & ND & ND & ND & $2655(28.4)$ & $2633(28.3)$ & $31(31)$ & $34(34)$ \\
\hline $\operatorname{BMI}\left(\mathrm{Kg} / \mathrm{m}^{2}\right)$ & $26 \cdot 9(4 \cdot 2)$ & $26 \cdot 7(4 \cdot 0)$ & $\begin{array}{c}23.7 \\
(15.6-43.4) \mathrm{c}\end{array}$ & $23.6(14.2-38.6)$ & $24.6 \pm 3.0$ & $24.9 \pm 3.2$ & ND & ND & $27(13-68)$ & $27(13-70)$ & ND & ND \\
\hline Body weight $(\mathrm{kg})$ & ND & ND & $63(35-104)$ & $62(36-109)$ & $\mathrm{ND}$ & ND & ND & ND & $80.0(28-174)$ & $80.0(29-180)$ & $\mathrm{ND}$ & ND \\
\hline Weight $<60 \mathrm{~kg}(\%)$ & $30(6)$ & $35(7)$ & $154(38.4)$ & $152(38.0)$ & ND & ND & ND & ND & $652(7.0)$ & $660(7.1)$ & ND & ND \\
\hline \multicolumn{13}{|l|}{ Risk factors } \\
\hline Diabetes mellitus (\%) & $150(30)$ & $146(29)$ & $154(38.4)$ & $124(31.0)$ & $116(29.0)$ & $100(25.0)$ & $58(29)$ & $42(21)$ & $2326(24.9)$ & $2336(25.1)$ & $42(42)$ & $39(39)$ \\
\hline Dyslipidemia (\%) & $325(65)$ & $323(65)$ & $314(78.3)$ & $289(72.3)$ & $208(52.0)$ & $194(48.5)$ & $88(44)$ & $74(37)$ & 4347 (46.6) & $4342(46.7)$ & $84(84)$ & $79(79)$ \\
\hline Hypertension (\%) & $365(73)$ & $362(73)$ & $305(76.1)$ & $290(72.5)$ & $223(55.8)$ & $193(48.2)$ & $122(61)$ & $116(58)$ & $6139(65.8)$ & $6044(65.1)$ & $79(79)$ & $82(82)$ \\
\hline Smoking (\%) & $62(13)$ & $67(14)$ & $151(37.7)$ & $157(39.3)$ & $146(36.5)$ & $139(34.8)$ & $116(58)$ & $124(62)$ & $3360(36.0)$ & $3318(35.7)$ & $37(37)$ & $41(41)$ \\
\hline \multicolumn{13}{|l|}{ Previous medical history } \\
\hline MI (\%) & $136(27)$ & $121(24)$ & $33(8.2)$ & $31(7.8)$ & $25(6.2)$ & $20(5.0)$ & $16(8)$ & $10(5)$ & $1900(20.4)$ & $1924(20.7)$ & $17(17)$ & $15(15)$ \\
\hline $\begin{array}{l}\text { Peripheral arterial disease } \\
(\%)\end{array}$ & $49(10)$ & $62(12)$ & $13(3.2)$ & $14(3.5)$ & $4(1.0)$ & $2(0.5)$ & $10(5)$ & $8(4)$ & $566(6.1)$ & $578(6.2)$ & $5(5)$ & $7(7)$ \\
\hline Congestive heart failure (\%) & ND & ND & $30(7.5)$ & $28(7.0)$ & $10(2.5)$ & $6(1.5)$ & ND & ND & $513(5.5)$ & $537(5.8)$ & $13(13)$ & $9(9)$ \\
\hline Angina pectoris (\%) & ND & ND & $102(25.4)$ & $110(27.5)$ & ND & ND & $114(57)$ & $104(52)$ & ND & ND & $40(40)$ & $36(36)$ \\
\hline $\begin{array}{c}\text { Atrial } \\
\text { fibrillation/flutter }(\%)\end{array}$ & ND & ND & ND & ND & ND & ND & $18(9)$ & $22(11)$ & ND & ND & ND & ND \\
\hline Non-hemorrhagic stroke (\%) & ND & ND & $27(6.7)$ & $28(7.0)$ & ND & ND & ND & ND & $353(3.8)$ & $369(4.0)$ & $11(11)$ & $10(10)$ \\
\hline Peptic ulcer disease (\%) & ND & ND & $37(9.2)$ & $37(9.3)$ & ND & ND & ND & ND & $\mathrm{ND}$ & ND & ND & ND \\
\hline Gastrointestinal bleeding (\%) & ND & ND & $6(1.5)$ & $7(1.8)$ & $1(0.2)$ & $0(0.0)$ & ND & ND & ND & ND & ND & ND \\
\hline Asthma (\%) & ND & ND & $12(3.0)$ & $14(3.5)$ & $12(3.0)$ & $3(0.8)$ & ND & ND & $267(2.9)$ & $265(2.9)$ & ND & ND \\
\hline $\begin{array}{l}\text { Dyspnea }(\%) \\
\text { CABG }(\%)\end{array}$ & $\begin{array}{l}\text { ND } \\
86(17)\end{array}$ & $\begin{array}{l}\text { ND } \\
84(17)\end{array}$ & $\begin{array}{l}32(8.0) \\
5(1.2)\end{array}$ & $\begin{array}{c}41(10.3) \\
1(0.3)\end{array}$ & $\begin{array}{l}\mathrm{ND} \\
4(1.0)\end{array}$ & $\begin{array}{c}\mathrm{ND} \\
3(0.8)\end{array}$ & $\begin{array}{l}\text { ND } \\
\text { ND }\end{array}$ & $\begin{array}{l}\text { ND } \\
\text { ND }\end{array}$ & $\begin{array}{l}1412(15.1) \\
532(5.7)\end{array}$ & $\begin{array}{c}1358(14.6) \\
574(6.2)\end{array}$ & $\begin{array}{l}\text { ND } \\
0(0)\end{array}$ & $\begin{array}{l}\text { ND } \\
0(0)\end{array}$ \\
\hline Ischaemic stroke (\%) & $25(5)$ & $22(4)$ & ND & ND & $24(6.0)$ & $16(4.0)$ & ND & ND & ND & ND & ND & ND \\
\hline $\begin{array}{c}\text { Transient ischaemic attack } \\
(\%)\end{array}$ & $38(8)$ & $37(7)$ & ND & ND & ND & ND & $32(16)$ & $34(17)$ & ND & ND & $16(16)$ & $14(14)$ \\
\hline
\end{tabular}


Table 2. Cont

\begin{tabular}{|c|c|c|c|c|c|c|c|c|c|c|c|c|}
\hline \multirow[b]{2}{*}{ Medication } & \multicolumn{2}{|c|}{ Gimbel et al., 2020 [14] } & \multicolumn{2}{|c|}{ Goto et al., 2015 [27] } & \multicolumn{2}{|c|}{ Park et al., 2019 [26] } & \multicolumn{2}{|c|}{ Tang et al., 2016 [25] } & \multicolumn{2}{|c|}{$\begin{array}{l}\text { Wallentin et al., } \\
2009[6]\end{array}$} & \multicolumn{2}{|c|}{ Wang and Wang 2016 [24] } \\
\hline & Ticagrelor & Clopidogrel & Ticagrelor & Clopidogrel & Ticagrelor & Clopidogrel & Ticagrelor & Clopidogrel & Ticagrelor & Clopidogrel & Ticagrelor & Clopidogrel \\
\hline Chronic renal disease (\%) & ND & ND & $18(4.5)$ & $20(5.0)$ & $6(1.5)$ & $1(0.2)$ & ND & $\mathrm{ND}$ & $379(4.1)$ & $406(4.4)$ & ND & ND \\
\hline Gout (\%) & ND & ND & $23(5.7)$ & $23(5.7)$ & $5(1.2)$ & $4(1.0)$ & ND & ND & $272(2.9)$ & $262(2.8)$ & ND & ND \\
\hline \multicolumn{13}{|l|}{ Diagnosis } \\
\hline NSTEMI (\%) & $424(86)$ & $423(86)$ & $66(16.5)$ & $74(18.5)$ & $148(37.0)$ & $155(38.8)$ & ND & ND & $4005(42.9)$ & $3950(42.5)$ & $44(44)$ & $47(47)$ \\
\hline STEMI (\%) & ND & ND & $205(51.1)$ & $210(52.5)$ & $170(42.5)$ & $156(39.0)$ & ND & ND & $3496(37.5)$ & $3530(38.0)$ & $37(37)$ & $32(32)$ \\
\hline Unstable angina $(\%)$ & $52(11)$ & $54(11)$ & 119 (29.7) & 109 (27.3) & $82(20.5)$ & $89(22.2)$ & ND & ND & $1549(16.6)$ & $1563(16.8)$ & $19(19)$ & $21(21)$ \\
\hline Positive troponin (\%) & ND & ND & 309 (77.1) & 298 (74.5) & $338(84.5)$ & $333(83.3)$ & ND & ND & $\begin{array}{c}7965 / 9333 \\
(85.3)\end{array}$ & $\begin{array}{c}7999 / 9291 \\
(86.1)\end{array}$ & ND & ND \\
\hline \multicolumn{13}{|l|}{ ECG findings } \\
\hline $\begin{array}{l}\text { Persistent ST-segment } \\
\text { elevation }(\%)\end{array}$ & ND & ND & $218(54.4)$ & $225(56.3)$ & ND & ND & ND & ND & 3497 (37.5) & $3511(37.8)$ & ND & ND \\
\hline ST-segment depression (\%) & ND & ND & $188(46.9)$ & $153(38.3)$ & ND & ND & ND & ND & $4730(50.7)$ & $4756(51.2)$ & ND & ND \\
\hline T-wave inversion (\%) & ND & ND & $142(35.4)$ & $126(31.5)$ & ND & ND & ND & ND & $2970(31.8)$ & $2975(32.0)$ & ND & ND \\
\hline \multicolumn{13}{|l|}{ Discharge medications } \\
\hline Vitamin K antagonist (\%) & $62(12)$ & $65(13)$ & $\mathrm{ND}$ & $\mathrm{ND}$ & ND & ND & $\mathrm{ND}$ & ND & $\mathrm{ND}$ & ND & ND & ND \\
\hline Proton pump inhibitor (\%) & $446(91)$ & $446(90)$ & 167 (41.6) & $175(43.8)$ & $12(3.0)$ & $8(2.0)$ & ND & ND & $4233(45.4)$ & $4128(44.4)$ & $31(31)$ & $33(33)$ \\
\hline$\beta$-blocker $(\%)$ & ND & ND & $40(10)$ & $44(11.1)$ & $275(68.8)$ & 297 (74.2) & 82 & 96 & $8339(89.3)$ & $8336(89.7)$ & $69(69)$ & $74(74)$ \\
\hline ACE inhibitor (\%) & ND & ND & $67(16.7)$ & $64(16.0)$ & ND & ND & ND & ND & $7090(76.0)$ & $6986(75.2)$ & $61(61)$ & $67(67)$ \\
\hline $\begin{array}{l}\text { Angiotensin receptor blocker } \\
(\%)\end{array}$ & ND & ND & $102(25.4)$ & $95(23.8)$ & $163(40.8)$ & $171(42.8)$ & ND & ND & $1143(12.2)$ & $1125(12.1)$ & & \\
\hline Statin $(\%)$ & ND & ND & 215 (53.6) & 205 (51.3) & 354 (88.5) & $369(92.2)$ & 198 & 199 & $8373(89.7)$ & $8289(89.2)$ & $83(83)$ & $79(79)$ \\
\hline Calcium channel blocker (\%) & ND & ND & $117(29.2)$ & $109(27.3)$ & $90(22.5)$ & $90(22.5)$ & ND & ND & $2769(29.7)$ & $2789(30.0)$ & $69(69)$ & $63(63)$ \\
\hline \multicolumn{13}{|l|}{ Procedure } \\
\hline Coronary angiography (\%) & $452(90)$ & $439(88$ & $385(96.0)$ & $378(95.4)$ & ND & ND & ND & ND & $7599(81.4)$ & $7571(81.5)$ & $86(86)$ & $83(83)$ \\
\hline PCI (\%) & $242(48)$ & $232(46)$ & $340(84.8)$ & 338 (84.5) & ND & ND & ND & ND & $5978(64.1)$ & $5999(64.6)$ & $75(75)$ & $71(71)$ \\
\hline BMS (\%) & $6(3)$ & $2(1)$ & ND & ND & ND & ND & ND & ND & $3921(42.0)$ & $3892(41.9)$ & ND & ND \\
\hline DES (\%) & $224(93)$ & $219(94)$ & ND & ND & ND & ND & ND & ND & $1719(18.4)$ & $1757(18.9)$ & ND & ND \\
\hline CABG (\%) & $87(17)$ & $78(16)$ & $9(2.2)$ & $3(0.8)$ & ND & ND & ND & ND & $931(10.0)$ & $968(10.4)$ & $0(0)$ & $0(0)$ \\
\hline
\end{tabular}

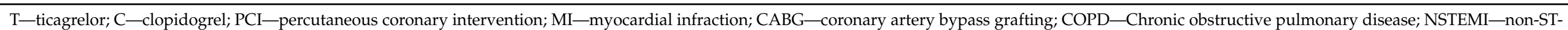
elevation myocardial infraction; STEMI—ST-elevation myocardial infraction; BMS— bare metal stent; DES— drug eluting stent; ND—no data. 


\subsection{Primary Outcomes: MACE, MI, ST and All-Cause Death}

Results of this analysis did not show any significant difference in the outcomes between ticagrelor and clopidogrel treatment for MACE (incidence: $9.23 \%$ vs. 10.57\%; $\mathrm{RR}=0.95,95 \% \mathrm{CI}=0.70-1.28, p=0.72$ ), $\mathrm{MI}$ (incidence: $5.40 \%$ vs. $6.23 \%$; $\mathrm{RR}=0.94$, $95 \% \mathrm{CI}=0.69-1.27, p=0.67$ ), ST (incidence: $2.33 \%$ vs. $3.17 \%$; RR $=0.61,95 \% \mathrm{CI}=0.32-1.17$, $p=0.13$ ), and all-cause death (incidence: $4.29 \%$ vs. $5.33 \%$; $R R=0.86,95 \% \mathrm{CI}=0.65-1.12$, $p=0.25)$ as shown in Figure 3.

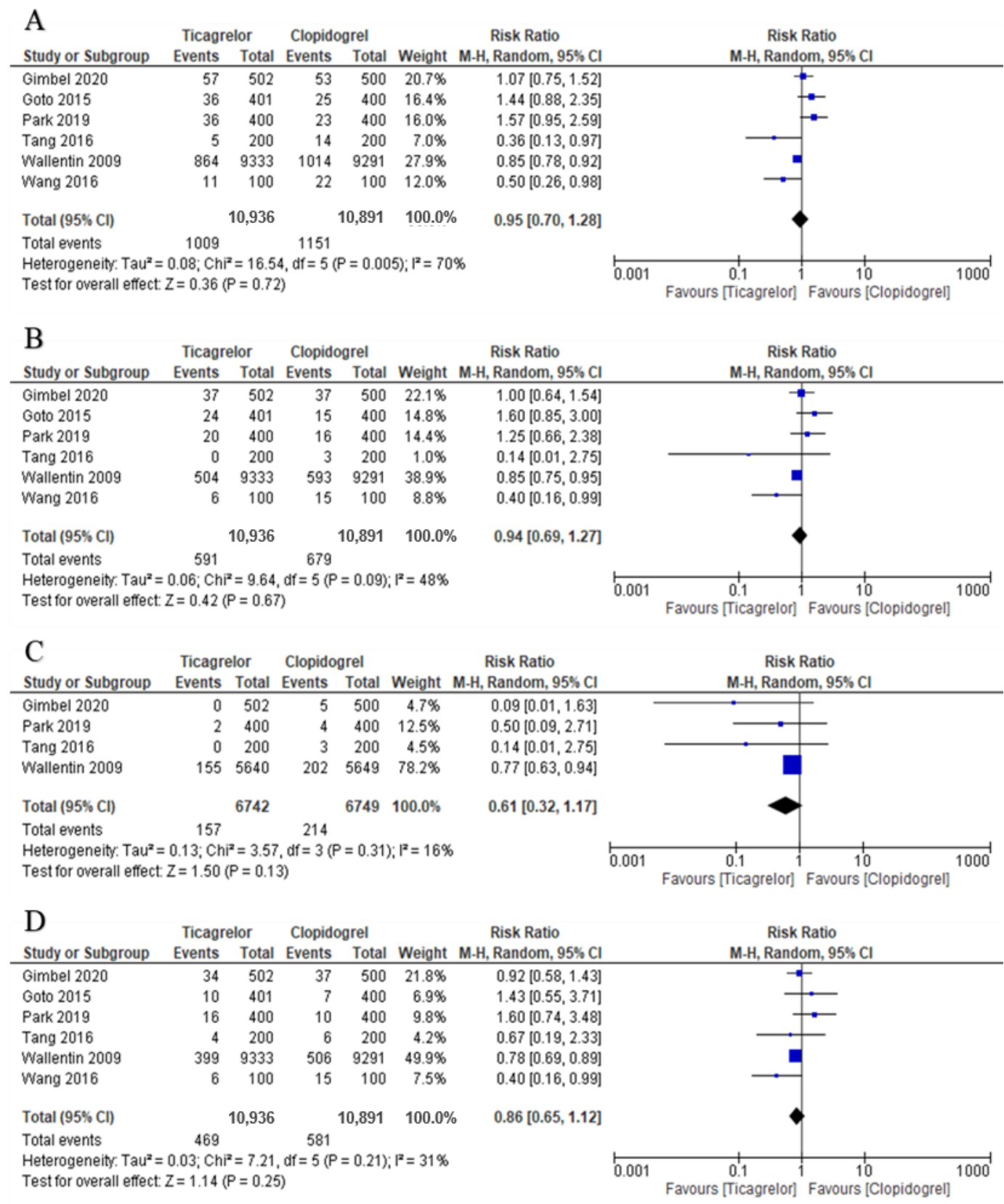

Figure 3. The forest plots for (A): MACE; (B): MI; (C): stent thrombosis; (D): all-cause mortality in elderly patients treated with Ticagrelor versus clopidogrel.

\subsection{Secondary Outcomes: Major Bleeding}

This meta-analysis did not show any significant difference between ticagrelor and clopidogrel for major bleeding (incidence: $9.98 \%$ vs. $9.33 \%$ : RR $=1.37,95 \% \mathrm{CI}=0.97-1.94$, $p=0.07$ ) (Figure 4). 


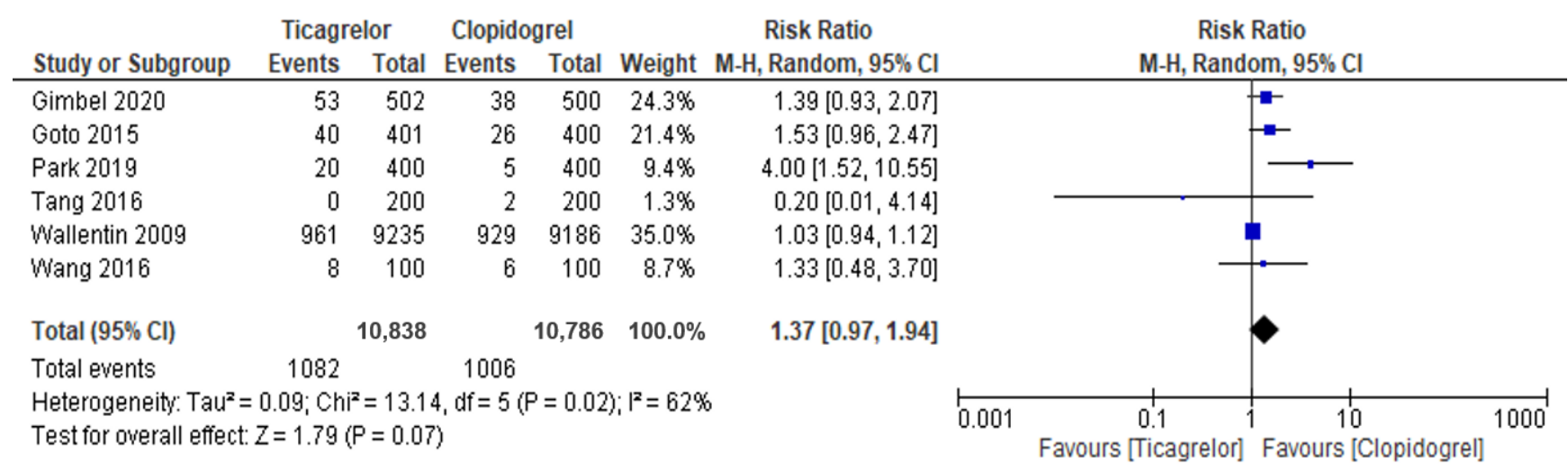

Figure 4. The forest plots for major bleeding in elderly patients treated with Ticagrelor versus clopidogrel.

\subsection{Sensitivity Analyses}

The sensitivity analysis results showed consistency in some of the studies when excluding studies one by one. Each time a new analysis was performed did not significantly impact the pooled results and conclusions of the study. However, the only difference was that the result representing ST was significant (incidence: $2.52 \%$ vs. $3.34 \%$; RR $=0.76$, $95 \% \mathrm{CI}=0.62-0.93, p=0.008)$ when we excluded the Gimbel et al. study; also, ST was significant (incidence: $0.18 \%$ vs. $1.09 \%$; $R R=0.28,95 \% C I=0.07-1.02, p=0.008$ ) when we excluded the Wallentin et al. study. In addition, major bleeding was significant (incidence: $7.55 \%$ vs. $4.81 \%$; $\mathrm{RR}=1.59,95 \% \mathrm{CI}=1.08-2.35, p=0.02$ ) when we excluded the Wallentin et al. study.

\section{Discussion}

We conducted a meta-analysis of RCTs to compare the effectiveness and safety of ticagrelor vs. clopidogrel in elderly patients with CHD. Ticagrelor's efficacy and safety have been similar to that of clopidogrel. Therefore, our comparison will focus more on the distinctive mechanisms of action of ticagrelor vs. clopidogrel. The mechanism of action of ticagrelor differs from clopidogrel in that clopidogrel acts irreversibly by blocking the P2Y12 receptor [28]. It is absorbed in the intestine, then metabolized by hepatic cytochrome P450 enzymes to active metabolites, and permanently binds to the P2Y12 receptor [29]. Ticagrelor, unlike clopidogrel, does not require activation of the metabolism. Thus, it is less affected by the variability associated with CYP polymorphisms in theory and thus produces a more antiplatelet effect [30-32]. Therefore, in elderly patients with low platelet aggregation, coagulation dysfunction, and other high bleeding risks, ticagrelor treatment must be used cautiously [26].

Compared to our findings, the therapeutic benefit of ticagrelor vs. clopidogrel was not significantly different between patients $\geq 75$ years old and those $<75$ years old, according to a substudy of the PLATO trial. Also, no increase in major bleeding events was observed with ticagrelor vs. clopidogrel in patients aged $\geq 75$ or $<75$ years (HR 1.02, 95\% CI $0.82-1.27$ ) or (HR 1.04, 95\% CI 0.94-1.15), respectively [12]. This is similar to our findings, which revealed that ticagrelor was not linked to a significant rate of major bleeding vs. clopidogrel.

In a study comparing ticagrelor and clopidogrel in Asian and non-Asian people with ACS, Kang et al., (2015) found no significant difference in efficacy between the two drugs. [33]. In addition, a recent meta-analysis found no significant difference in effectiveness outcomes between potent P2Y12 (ticagrelor and prasugrel) inhibitors and clopidogrel, even though P2Y12 inhibitors were linked to significant bleeding, posing a risk to older patients [34]. In addition, a meta-analysis of the results of potent P2Y12 inhibitors vs. clopidogrel in elderly ACS patients found that potent P2Y12 inhibitors were more effective in reducing CV death and MI. However, they were not safe and increased bleeding risk compared with clopidogrel [35]. But their study included both ticagrelor and 
prasugrel, which would not give definitive results that strictly correlate with the use of ticagrelor vs. clopidogrel.

According to one study of elderly ACS patients using DAPT, ischemic events are more common in the acute phase, while bleeding events are more common in the late phase [36]. Therefore, patients are recommended to take aspirin with ticagrelor in the acute phase (during the first months of injury) followed by aspirin and clopidogrel in the late phase. It may be considered an effective and practical strategy to reduce ischemic and bleeding events in elderly patients [37]. This recommendation is supported by several studies where ticagrelor was switched to clopidogrel in ACS patients undergoing PCI [38-40]. Still, the effectiveness and safety of this switching require more studies to determine the appropriate time for switching.

\section{Limitations}

The meta-analysis contains some limitations. The hypotheses, inclusion and exclusion criteria, identification of elderly patients, and endpoints of the trials included in our study were slightly different. Furthermore, the dosages of the medications utilized were not considered. Finally, the quality of the literature included was variable. Therefore, this study has limited clinical guidance and is for reference only to clinicians, and its results should be considered exploratory. Further evidence-based studies are needed to verify the effectiveness and safety of ticagrelor vs. clopidogrel in elderly CHD patients.

\section{Conclusions}

This study showed ticagrelor is not significantly more efficacious than clopidogrel in elderly patients with CHD. In addition, it was not associated with significant major bleeding compared to clopidogrel.

Author Contributions: Conceptualization, M.A.A., B.I. and A.S.; methodology, M.A.A., B.I. and A.S.; formal analysis, M.A.A., B.I., A.S. and I.Y.; resources, M.A.A.; writing—original draft preparation, M.A.A.; writing-review and editing, N.A.A.D., I.Y., M.L.N., M.A.S.A.K. and D.A.M.N.; visualization, M.A.A., A.S. and B.I.; supervision, B.I. and A.S. All authors have read and agreed to the published version of the manuscript.

Funding: This research received no external funding.

Acknowledgments: The authors would like to express their immense gratitude to Ahmad Mustakim Mohamad, School of Distance Education, Universiti Sains Malaysia for their statistical support.

Conflicts of Interest: The authors declare no conflict of interest.

\section{References}

1. Roth, G.A.; Mensah, G.A.; Johnson, C.O.; Addolorato, G.; Ammirati, E.; Baddour, L.M.; Barengo, N.C.; Beaton, A.Z.; Benjamin, E.J.; Benziger, C.P. Global burden of cardiovascular diseases and risk factors, 1990-2019: Update from the GBD 2019 study. J. Am. Coll. Cardiol. 2020, 76, 2982-3021. [CrossRef]

2. Roth, G.A.; Abate, D.; Abate, K.H.; Abay, S.M.; Abbafati, C.; Abbasi, N.; Abbastabar, H.; Abd-Allah, F.; Abdela, J.; Abdelalim, A. Global, regional, and national age-sex-specific mortality for 282 causes of death in 195 countries and territories, 1980-2017: A systematic analysis for the Global Burden of Disease Study 2017. Lancet 2018, 392, 1736-1788. [CrossRef]

3. Roth, G.A.; Forouzanfar, M.H.; Moran, A.E.; Barber, R.; Nguyen, G.; Feigin, V.L.; Naghavi, M.; Mensah, G.A.; Murray, C.J. Demographic and epidemiologic drivers of global cardiovascular mortality. N. Engl. J. Med. 2015, 372, 1333-1341. [CrossRef]

4. Fox, K.A.; Dabbous, O.H.; Goldberg, R.J.; Pieper, K.S.; Eagle, K.A.; Van de Werf, F.; Avezum, Á.; Goodman, S.G.; Flather, M.D.; Anderson, F.A. Prediction of risk of death and myocardial infarction in the six months after presentation with acute coronary syndrome: Prospective multinational observational study (GRACE). BMJ 2006, 333, 1091. [CrossRef] [PubMed]

5. Mehran, R.; Pocock, S.J.; Nikolsky, E.; Clayton, T.; Dangas, G.D.; Kirtane, A.J.; Parise, H.; Fahy, M.; Manoukian, S.V.; Feit, F. A risk score to predict bleeding in patients with acute coronary syndromes. J. Am. Coll. Cardiol. 2010, 55, 2556-2566. [CrossRef] [PubMed]

6. Wallentin, L.; Becker, R.C.; Budaj, A.; Cannon, C.P.; Emanuelsson, H.; Held, C.; Horrow, J.; Husted, S.; James, S.; Katus, H. Ticagrelor versus clopidogrel in patients with acute coronary syndromes. N. Engl. J. Med. 2009, 361, 1045-1057. [CrossRef] [PubMed] 
7. Valgimigli, M.; Bueno, H.; Byrne, R.A.; Collet, J.-P.; Costa, F.; Jeppsson, A.; Jüni, P.; Kastrati, A.; Kolh, P.; Mauri, L. 2017 ESC focused update on dual antiplatelet therapy in coronary artery disease developed in collaboration with EACTS. Eur. J. Cardio-Thorac. Surg. 2018, 53, 34-78. [CrossRef] [PubMed]

8. Dhillon, S. Ticagrelor: A review of its use in adults with acute coronary syndromes. Am. J. Cardiovasc. Drugs 2015, 15, 51-68. [CrossRef]

9. Akkaif, M.A.; Daud, N.A.A.; Sha'aban, A.; Ng, M.L.; Sk Abdul Kader, M.A.; Noor, D.A.M.; Ibrahim, B. The Role of Genetic Polymorphism and Other Factors on Clopidogrel Resistance (CR) in an Asian Population with Coronary Heart Disease (CHD). Molecules 2021, 26, 1987. [CrossRef]

10. Yoon, H.Y.; Lee, N.; Seong, J.M.; Gwak, H.S. Efficacy and safety of clopidogrel versus prasugrel and ticagrelor for coronary artery disease treatment in patients with CYP2C19 LoF alleles: A systemic review and meta-analysis. Br. J. Clin. Pharmacol. 2020, 86, 1489-1498. [CrossRef]

11. Biswas, M.; Kali, M.S.K.; Biswas, T.K.; Ibrahim, B. Risk of major adverse cardiovascular events of CYP2C19 loss-of-function genotype guided prasugrel/ticagrelor vs. clopidogrel therapy for acute coronary syndrome patients undergoing percutaneous coronary intervention: A meta-analysis. Platelets 2021, 32, 591-600. [CrossRef]

12. Husted, S.; James, S.; Becker, R.C.; Horrow, J.; Katus, H.; Storey, R.F.; Cannon, C.P.; Heras, M.; Lopes, R.D.; Morais, J. Ticagrelor versus clopidogrel in elderly patients with acute coronary syndromes: A substudy from the prospective randomized PLATelet inhibition and patient Outcomes (PLATO) trial. Circ. Cardiovasc. Qual. Outcomes 2012, 5, 680-688. [CrossRef]

13. Avezum, A.; Makdisse, M.; Spencer, F.; Gore, J.M.; Fox, K.A.; Montalescot, G.; Eagle, K.A.; White, K.; Mehta, R.H.; Knobel, E. Impact of age on management and outcome of acute coronary syndrome: Observations from the Global Registry of Acute Coronary Events (GRACE). Am. Heart J. 2005, 149, 67-73. [CrossRef]

14. Gimbel, M.; Qaderdan, K.; Willemsen, L.; Hermanides, R.; Bergmeijer, T.; de Vrey, E.; Heestermans, T.; Gin, M.T.J.; Waalewijn, R.; Hofma, S. Clopidogrel versus ticagrelor or prasugrel in patients aged 70 years or older with non-ST-elevation acute coronary syndrome (POPular AGE): The randomised, open-label, non-inferiority trial. Lancet 2020, 395, 1374-1381. [CrossRef]

15. Szummer, K.; Montez-Rath, M.E.; Alfredsson, J.; Erlinge, D.; Lindahl, B.; Hofmann, R.; Ravn-Fischer, A.; Svensson, P.; Jernberg, T. Comparison between ticagrelor and clopidogrel in elderly patients with an acute coronary syndrome: Insights from the SWEDEHEART registry. Circulation 2020, 142, 1700-1708. [CrossRef] [PubMed]

16. Liberati, A.; Altman, D.G.; Tetzlaff, J.; Mulrow, C.; Gøtzsche, P.C.; Ioannidis, J.P.; Clarke, M.; Devereaux, P.J.; Kleijnen, J.; Moher, D. The PRISMA statement for reporting systematic reviews and meta-analyses of studies that evaluate health care interventions: Explanation and elaboration. J. Clin. Epidemiol. 2009, 62, e1-e34. [CrossRef] [PubMed]

17. Guidelines, E.C.f.P.; Bax, J.J.; Baumgartner, H.; Ceconi, C.; Dean, V.; Deaton, C.; Fagard, R.; Funck-Brentano, C.; Hasdai, D.; Hoes, A. Third universal definition of myocardial infarction. J. Am. Coll. Cardiol. 2012, 60, 1581-1598.

18. Kikkert, W.J.; van Geloven, N.; van der Laan, M.H.; Vis, M.M.; Baan, J.; Koch, K.T.; Peters, R.J.; de Winter, R.J.; Piek, J.J.; Tijssen, J.G. The prognostic value of bleeding academic research consortium (BARC)-defined bleeding complications in ST-segment elevation myocardial infarction: A comparison with the TIMI (Thrombolysis In Myocardial Infarction), GUSTO (Global Utilization of Streptokinase and Tissue Plasminogen Activator for Occluded Coronary Arteries), and ISTH (International Society on Thrombosis and Haemostasis) bleeding classifications. J. Am. Coll. Cardiol. 2014, 63, 1866-1875.

19. Sterne, J.A.; Savović, J.; Page, M.J.; Elbers, R.G.; Blencowe, N.S.; Boutron, I.; Cates, C.J.; Cheng, H.-Y.; Corbett, M.S.; Eldridge, S.M. RoB 2: A revised tool for assessing risk of bias in randomised trials. BMJ 2019, 366. [CrossRef] [PubMed]

20. Stare, J.; Maucort-Boulch, D. Odds ratio, hazard ratio and relative risk. Metodoloski Zv. 2016, 13, 59.

21. Higgins, J.P.; Thompson, S.G.; Deeks, J.J.; Altman, D.G. Measuring inconsistency in meta-analyses. BMJ 2003, 327, 557-560. [CrossRef] [PubMed]

22. Sterne, J.A.; Sutton, A.J.; Ioannidis, J.P.; Terrin, N.; Jones, D.R.; Lau, J.; Carpenter, J.; Rücker, G.; Harbord, R.M.; Schmid, C.H. Recommendations for examining and interpreting funnel plot asymmetry in meta-analyses of randomised controlled trials. BMJ 2011, 343. [CrossRef] [PubMed]

23. Guan, W.; Lu, H.; Yang, K. Choosing between ticagrelor and clopidogrel following percutaneous coronary intervention: A systematic review and Meta-Analysis (2007-2017). Medicine 2018, 97, e12978. [CrossRef]

24. Wang, H.; Wang, X. Efficacy and safety outcomes of ticagrelor compared with clopidogrel in elderly Chinese patients with acute coronary syndrome. Ther. Clin. Risk Manag. 2016, 12, 1101. [CrossRef]

25. Tang, X.; Li, R.; Jing, Q.; Wang, Q.; Liu, P.; Zhang, P.; Liu, Y. Assessment of ticagrelor versus clopidogrel treatment in patients with ST-elevation myocardial infarction undergoing primary percutaneous coronary intervention. J. Cardiovasc. Pharmacol. 2016, 68, 115-120. [CrossRef]

26. Park, D.-W.; Kwon, O.; Jang, J.-S.; Yun, S.-C.; Park, H.; Kang, D.-Y.; Ahn, J.-M.; Lee, P.H.; Lee, S.-W.; Park, S.-W. Clinically significant bleeding with ticagrelor versus clopidogrel in Korean patients with acute coronary syndromes intended for invasive management: A randomized clinical trial. Circulation 2019, 140, 1865-1877. [CrossRef]

27. Goto, S.; Huang, C.-H.; Park, S.-J.; Emanuelsson, H.; Kimura, T. Ticagrelor vs. clopidogrel in Japanese, Korean and Taiwanese patients with acute coronary syndrome-randomized, double-blind, phase III PHILO study. Circ. J. 2015, 79, 2452-2460. [CrossRef]

28. Savi, P.; Nurden, P.; Nurden, A.; Levy-Toledano, S.; Herbert, J.-M. Clopidogrel: A review of its mechanism of action. Platelets 1998, 9, 251-255. [CrossRef] 
29. Wallentin, L. P2Y12 inhibitors: Differences in properties and mechanisms of action and potential consequences for clinical use. Eur. Heart J. 2009, 30, 1964-1977. [CrossRef] [PubMed]

30. Birkeland, K.; Parra, D.; Rosenstein, R. Antiplatelet therapy in acute coronary syndromes: Focus on ticagrelor. J. Blood Med. 2010, 1, 197.

31. Akkaif, M.A.; Ng, M.L.; Kader, M.A.S.A.; Daud, N.A.A.; Sha'aban, A.; Ibrahim, B. A review of the effects of ticagrelor on adenosine concentration and its clinical significance. Pharmacol. Rep. 2021, 1-14. [CrossRef]

32. Akkaif, M.A.; Sha'aban, A.; Daud, N.A.A.; Ng, M.L.; Ibrahim, B. Investigate the Strategy of Using Pharmacogenetics and Pharmacometabonomics to the Personalization of Ticagrelor Antiplatelet Therapy. Syst. Rev. Pharm. 2020, 11, $1100-1107$.

33. Kang, H.-J.; Clare, R.M.; Gao, R.; Held, C.; Himmelmann, A.; James, S.K.; Lim, S.T.; Santoso, A.; Yu, C.-M.; Wallentin, L. Ticagrelor versus clopidogrel in Asian patients with acute coronary syndrome: A retrospective analysis from the Platelet Inhibition and Patient Outcomes (PLATO) Trial. Am. Heart J. 2015, 169, 899-905.e891. [CrossRef] [PubMed]

34. Fujisaki, T.; Kuno, T.; Ando, T.; Briasoulis, A.; Takagi, H.; Bangalore, S. Potent P2Y12 Inhibitors versus Clopidogrel in Elderly Patients with Acute Coronary Syndrome: Systematic Review and Meta-Analysis: P2Y12 inhibitors and elderly patients with ACS. Am. Heart J. 2021, 237, 34-44. [CrossRef] [PubMed]

35. Tarantini, G.; Ueshima, D.; D'Amico, G.; Masiero, G.; Musumeci, G.; Stone, G.W.; Brener, S.J. Efficacy and safety of potent platelet P2Y12 receptor inhibitors in elderly versus nonelderly patients with acute coronary syndrome: A systematic review and meta-analysis. Am. Heart J. 2018, 195, 78-85. [CrossRef] [PubMed]

36. Crimi, G.; Morici, N.; Ferrario, M.; Ferri, L.A.; Piatti, L.; Grosseto, D.; Cacucci, M.; Mandurino Mirizzi, A.; Toso, A.; Piscione, F. Time course of ischemic and bleeding burden in elderly patients with acute coronary syndromes randomized to low-dose prasugrel or clopidogrel. J. Am. Heart Assoc. 2019, 8, e010956. [CrossRef] [PubMed]

37. Cuisset, T.; Deharo, P.; Quilici, J.; Johnson, T.W.; Deffarges, S.; Bassez, C.; Bonnet, G.; Fourcade, L.; Mouret, J.P.; Lambert, M. Benefit of switching dual antiplatelet therapy after acute coronary syndrome: The TOPIC (timing of platelet inhibition after acute coronary syndrome) randomized study. Eur. Heart J. 2017, 38, 3070-3078. [CrossRef]

38. Franchi, F.; Rollini, F.; Rivas Rios, J.; Rivas, A.; Agarwal, M.; Kureti, M.; Nagaraju, D.; Wali, M.; Shaikh, Z.; Briceno, M. Pharmacodynamic effects of switching from ticagrelor to clopidogrel in patients with coronary artery disease: Results of the SWAP-4 study. Circulation 2018, 137, 2450-2462. [CrossRef]

39. Li, X.Y.; Su, G.H.; Wang, G.X.; Hu, H.Y.; Fan, C.J. Switching from ticagrelor to clopidogrel in patients with ST-segment elevation myocardial infarction undergoing successful percutaneous coronary intervention in real-world China: Occurrences, reasons, and long-term clinical outcomes. Clin. Cardiol. 2018, 41, 1446-1454. [CrossRef]

40. Pourdjabbar, A.; Hibbert, B.; Chong, A.-Y.; Le May, M.R.; Labinaz, M.; Simard, T.; Ramirez, F.D.; Lugomirski, P.; Maze, R.; Froeschl, M. A randomised study for optimising crossover from ticagrelor to clopidogrel in patients with acute coronary syndrome. Thromb. Haemost. 2017, 117, 303-310. [CrossRef] 